\title{
Combined hepatocellular-cholangiocarcinoma containing the cells of hepatocellular cancer and bile duct cancer
}

\author{
Oleksandr Legkiy ${ }^{1}$, Anna Ćwierz ${ }^{2}$, Joanna Wysocka ${ }^{3}$, Andrzej L. Komorowski ${ }^{1}$
}

Combined hepatocellular-cholangiocarcinoma (cHCC-CC) is a rare $(<1 \%)$ primary liver cancer which contains elements of hepatocellular cancer (HCC) and bile duct cancer (CC). On account of the difficulties in reaching preoperative diagnosis, it is frequently diagnosed only after resection is performed. A surgical resection of the liver is the treatment of choice, whilst a liver transplant is a rarely implemented option. The prognosis of CHCC-CC tumours is better than in CC but worse than in HCC. This paper presents the case report of a 62-year-old man undergoing a left hemihepatectomy for a cHCC-CC liver tumour.

NOWOTWORY J Oncol 2018; 68, 2: 92-94

Key words: HCC-CC, liver, hemihepatectomy

\section{Introduction}

The simultaneous occurrence of hepatocellular cancer (HCC) and bile duct cancer (CC) within a liver tumour is regarded as a separate clinical entity (cHCC-CC — combined hepatocellular-cholangiocarcinoma). This type of cancer (cHCC-CC) was described for the first time in 1949 by Allen and Lis [1]. The CHCC-CC tumour is a rare malignant hepatic tumour and accounts for less than $1 \%$ of all primary malignant liver cancers [2]. This type of cancer is characterised by fast growth and a significant metastatic potential. It occurs more frequently in men than in women: 14:1 [3], usually in the seventh decade of life $[2,4]$. The factors which might affect the development of this type of cancer comprise an infection with $\mathrm{HCV}, \mathrm{HBV}$ and liver cirrhosis $[3,5,7]$. On the basis of clinical data and diagnostic imaging, a preoperative diagnosis is difficult [6]. The prognosis depends on the stage and is generally better than in CC but worse than in HCC. [2].

This paper presents the case report of a 62-year-old male patient undergoing a left hemihepatectomy for a cHCC-CC liver tumour.

\section{The site's observations}

A 62-year-old male patient, without a history of oncological diseases was admitted to the Department of Surgical Oncology on account of a liver cancer diagnosed accidentally in an ultrasound examination. The abdominal ultrasound revealed a lesion with mixed echogenicity in the left (in accordance with surgical anatomy) liver lobe, measuring $77 \times 60 \mathrm{~mm}$. The lesion, involving segments: II, III, IVa and IVb was shown in the PET examination with SUV max (standard uptake value) measuring 9.8 (Fig. 1). Neither CT not Pet revealed any other disease foci, in particular - no involvement of the lymph nodes was found. The biochemistry findings were the following: CA - 19.9-55.85 $\mu / \mathrm{ml}$, CEA - $8.46 \mathrm{ng} / \mathrm{ml}$, AFP - $20.94 \mathrm{ng} / \mathrm{ml}$, ALAT - $860 \mu / \mathrm{l}$. The remaining tests (including $\mathrm{HBV}$ and $\mathrm{HCV}$ ) were unremarkable.

The patient was qualified for surgery. During the intervention, a tumour was found in the left hepatic lobe, involving the segments: II, III, IVa and IVb. A left hemihepatectomy with lymphangiectomy of the hepatoduodenal ligament and the splenic vein was performed The histo-

${ }^{1}$ Department of Surgical Oncology, Maria Skłodowska-Curie Memorial Cancer Centre and Institute of Oncology, Kraków Branch, Poland

${ }^{2}$ Department of Diagnostic Imaging, Ludwik Rydygier Memorial Specialised Hospital, Kraków, Poland

${ }^{3}$ Department of Pathology, Maria Skłodowska-Curie Memorial Cancer Centre and Institute of Oncology, Kraków Branch, Poland 


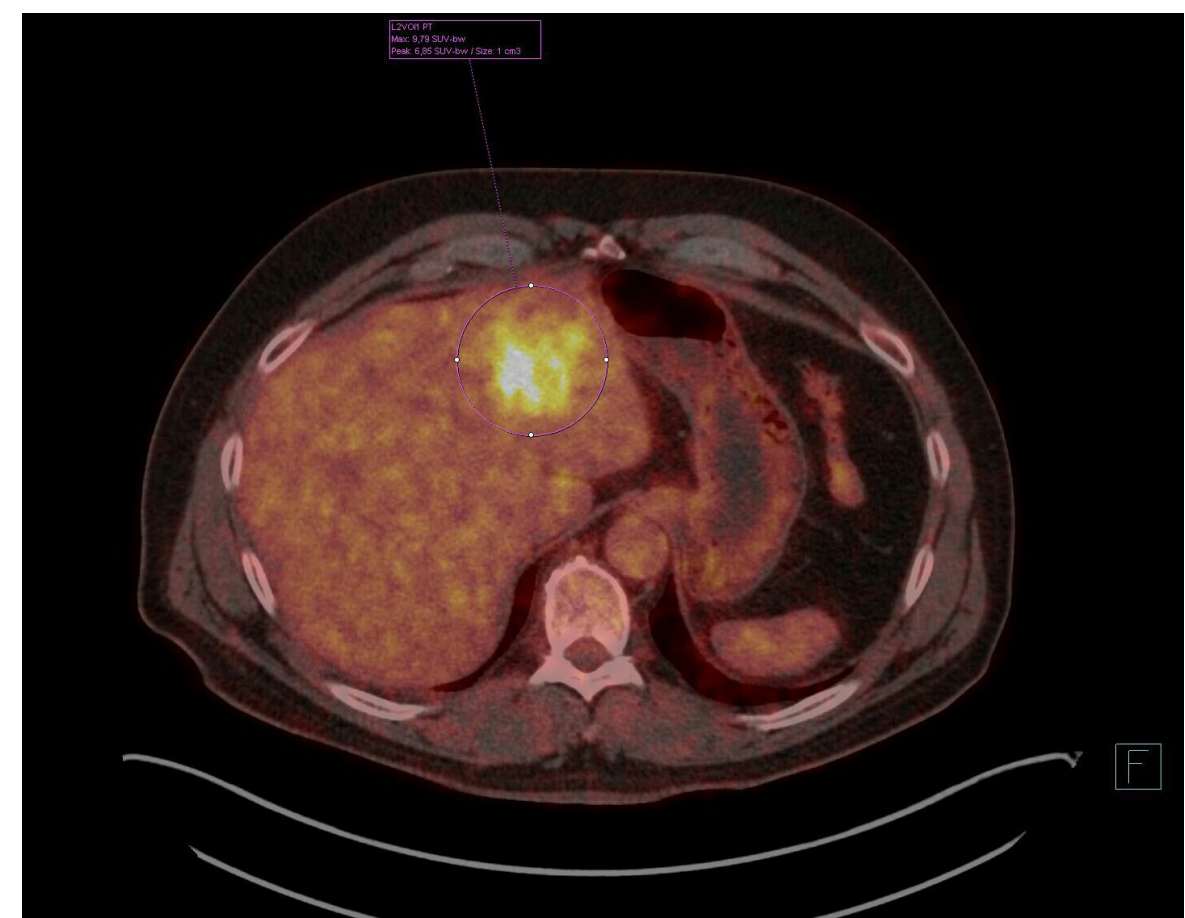

Figure 1. The image of the tumour of the left liver love in PET imaging

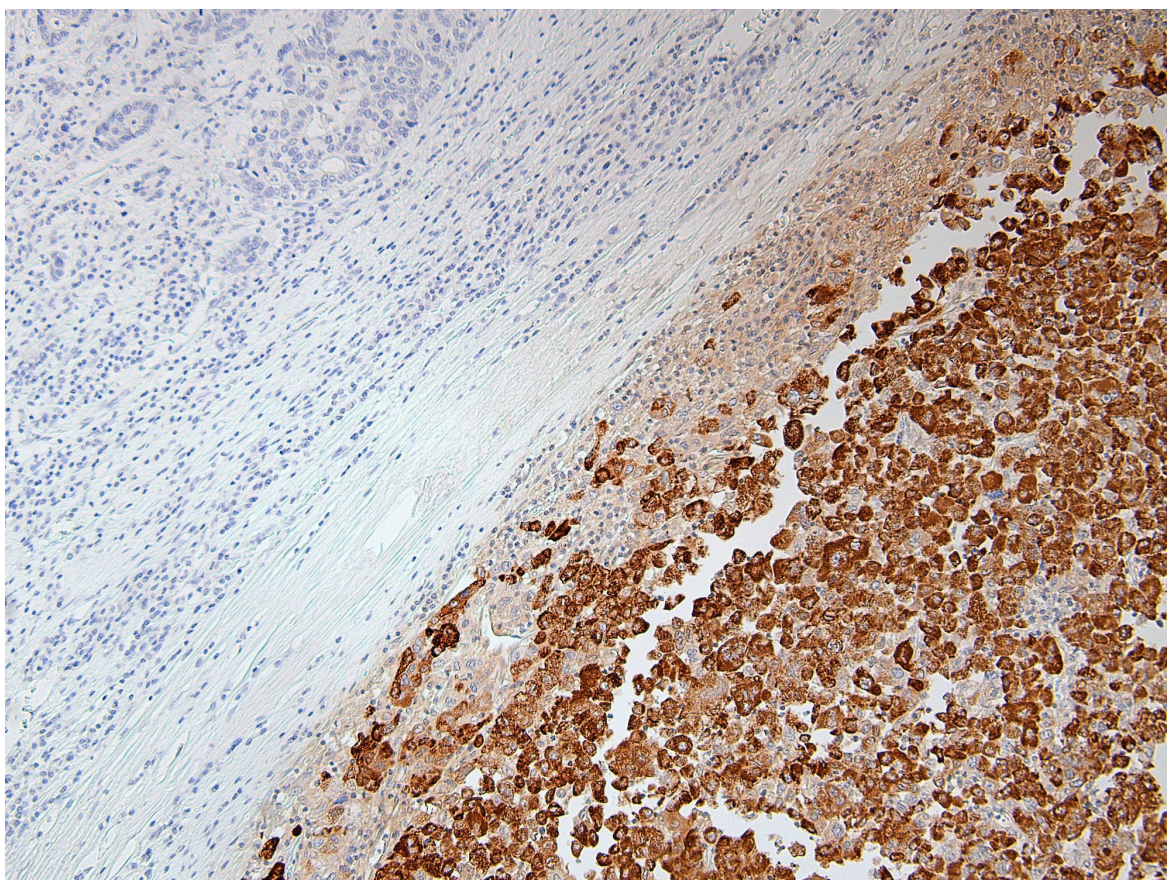

Figure 2. In the bottom right corner the architecture of hepatocellular carcinoma is seen - the immunohistochemical reaction to the presence of Hep-Par-1 in the cancer cells is positive; in the top left corner the architecture of bile duct cancer is visible - the reaction to the presence of Hep-Par-1 is negative; magnification $\times 100$

pathological assessment revealed the architecture of two types of tumour: HCC and CC with a dominating histological component of HCC (Fig. 2) and two lymph nodes without cancer lesions. Both in the primary pathological report and in the microscopic assessment of the tumours performed later on (for the purposes of this publication), the differentiation grade of the hepatocellular carcinoma (HCC) was defined as 3, whilst that of the bile duct cancer (CC) was 2 . Additionally, in the case of both types of architecture, the signs of vascular involvement were found (LVI +).

The post-operative course was uneventful. The patient was discharged home on the $6^{\text {th }}$ day post-op. Six months after the surgery, a multifocal recurrence was found in the right hepatic lobe and verified with a transdermal 
core needle biopsy as CC. The patient was qualified for further surgery and chemotherapy with gemcitabine and cisplatin. The chemotherapy was discontinued on account of a myocardial infarction which had to be treated with an angioplasty. Eight months after the surgery, the patient lives with a symptomatic disease.

\section{Discussion}

The presence of a tumour with an architecture invoIving both hepatocellular carcinoma and bile duct carcinoma (cHCC-CC) in the liver is quite rare, accounting for $<1 \%$ of all primary liver cancers [2]. It is more prevalent in Caucasian males and among people above 65 years of age. The pathogenic factors affecting the development of this type of tumour have not been definitively established. The publications mention an infection with hepatotropic viruses (infections with HCV in 10-12\% [5,8], with HBV in $54 \%$ patients [5]) and liver cirrhosis occurring in 54\% [5] as the predisposing factors for the development of CHCC-CC.

On the basis of the analysis of the data from the SEER database (The Surveillance, Epidemiology, and End Results) covering 52825 patients with primary liver cancers, including 465 patients with $\mathrm{CHCC}-\mathrm{CC}$, it was found that within this group of patients, a significantly worse prognosis concerns people of black race, with a significant progression of the disease and with the presence of tumours measuring from 5 to $10 \mathrm{~cm}$ in diameter. Favourable prognostic factors affecting the improvement of prognoses in the multivariate analysis were diagnoses made after 1995 and extensive surgical intervention (tumour resection or liver transplant) [2]. In the analysis made by et al, adverse prognostic factors in CHCC-CC patients included: the presence of numerous tumours, lymph node involvement and the infiltration of the porta vein or hepatic vein [8].

In comparison with other primary liver cancers, CHCC-CC can be characterised with medium prognosis. Overall 5 -year survival in patients with this type of cancer is about $10.5 \%$, whilst in patients with CC it is $5.7 \%$, and in patients with $\mathrm{HCC}-21 \%$ [2]. A radical resection of the tumour with CHCC-CC architecture allows for obtaining a 5-year survival rate reaching $28 \%$ [2], which is significantly worse than in the case of a tumour resection in HCC tumour architecture, where the overall 5-year survival makes up 42.3\% [9]. Overall 5-year survival after a liver transplant leads to $41.1 \%$ survival for CHCC-CC patients, whereas for CC tumours 3-year survival is obtained solely by $14.8 \%$ patients [10]. The results of the application of local ablation techniques in the treatment of cHCC-CC are poor [2].
Benefits in the use of chemotherapy in the treatment of cHCC-CC tumours have not been completely understood. The publications mention some isolated cases of the application of chemotherapy (gemcitabine and cisplatin) in the dissemination stage of the cancer [11]. The use of other techniques, such as radiotherapy and transdermal ethanol injections, did not lead to any satisfactory results [12].

Within the post-operative follow-up, a periodic ultrasound and $\mathrm{Ct}$ evaluation is used as well as the measurement of the levels of AFP and CA19-9 in blood serum [12].

Patients with primary liver tumours of the CHCC-CC type, in comparison with other primary liver cancers, are characterised with moderate prognoses. The treatment of choice comprises extensive resection or — more rarely — a liver transplant.

\section{Conflict of interest: none declared}

\section{Oleksandr Legkiy, MD}

Maria Skłodowska-Curie Memorial Cancer Centre and Institute of Oncology

Kraków Branch

Department of Surgical Oncology

ul. Garncarska 11, 31-115 Kraków, Poland

e-mail.oleksandr.legkiy@gmail.com

Received: 3 Apr 2018

Accepted: 5 Jun 2018

\section{References}

1. Allen RA, Lisa JR. Combined liver cell and bile duct carcinoma. Am J Pathol 1949; 25: 647-655.

2. Garancini M, Goffredo P, Pagni F et al. Combined hepatocellular-chorangiocarcinoma: a population-level analysis of an uncommon primary liver tumor. Liver Transpl 2014; 20: 952-959.

3. Maximin S, Ganeshan DM, Shanbhogue AK et al. Current update on combined hepatocellular-cholangiocarcinoma. EurJRadiol Open 2014; 1:40-48.

4. Yeh M. Pathology of combined hepatocellular-cholangiocarcinoma. J Gastroenterol Hepatol 2010; 25: 1485-1492.

5. Koh KC, Lee H, Choi MS et al. Clinicopathologic features and prognosis of combined hepatocellular cholangiocarcinoma. Am J Surg 2005; 189: 120-125.

6. Kassahun W, Haus J. Management of combined hepatocellular and cholangiocarcinoima. Int J Clin Pract 2008; 62: 1271-1278.

7. Watanabe T, Sakata J, Ishikawa T et al. Synchronous development of $\mathrm{HCC}$ and CCC in the same subsegment of the liver in a patient with type C liver cirrhosis. World J Hepatol 2009; 1: 103-109.

8. Chan AC, Lo CM, Ng IO et al. Liver transplantation for combined hepatocellular cholangiocarcinoma. Asian J Surg 2007; 30: 143-146.

9. Grąt M, Hołówko W, Grzegorczyk K et al. Long-term results of liver resection in the treatment of patients with hepatocellular carcinoma. Pol Przegl Chir 2011; 83: 319-324.

10. Patkowski W, Stankiewicz R, Grat M et al. Poor outcomes after liver transplantation in patients with incidental cholangiocarcinoma irrespective of tumor localization. Transplant Proc 2014; 46: 2774-2776.

11. Chi M, Mikhitarian K, Shi C et al. Management of combined hepatocellular-cholangiocarcinoma: a case report and lieterature review. Gastrointest Cancer Res 2012; 5: 199-202.

12. O'Connor K, Walsh JC, Schaeffer DF. Combined hepatocellular-cholangiocarcinoma (cHCC-CC): a distinct entity. Ann Hepatol 2014; 13: 317-322. 